MS9. Pharmaceutical

crystallography and drug design

Chairs: David Brown, Andreas Heine

\section{MS9-P1 Understanding nanoscale order in amorphous pharmaceuticals}

Konstantin B. Borisenko ${ }^{1}$, Wanjing Li $^{2}$, Graham Buckton ${ }^{2}$, Andrew Stewart ${ }^{3}$, Angus I. Kirkland ${ }^{1}$

1. Department of Materials, University of Oxford, Parks Road, Oxford OX1 3PH, UK.

2. School of Pharmacy, Faculty of Life Sciences, University College London, 29-39 Brunswick Square, London, WC1N 1AX, UK.

3. Department of Physics and Energy, University of Limerick, Limerick, Ireland.

email: konstantin.borisenko@materials.ox.ac.uk

According to differential scanning calorimetry spray-dried pharmaceutical formulations of indomethacin and polyvinylpyrrolidone form amorphous solid solution. These formulations also appear amorphous when examined by X-ray diffraction. However, these materials still possess nanocrystalline order as revealed by nanoscale electron diffraction. The structure of the nanocrystals of indomethacine appear to represent a range of the known bulk crystalline structures as well as new phases. We discuss implications of nanoscale organisation in amorphous pharmaceuticals for building reliable structure - properties correlations in such materials.

Keywords: amorphous pharmaceuticals, electron diffraction, nanoscale order

\section{MS9-P2 Pharmaceutical drug discovery at the IMCA-CAT Advanced Photon Source user facility}

Anne Mulichak ${ }^{1}$, Anne Mulichak ${ }^{1}$, Kevin P. Battaile ${ }^{1}$, Joe Digilio $^{1}$, J. Lewis Muir ${ }^{1}$, Eric Zoellner ${ }^{1}$, Lisa J. Keefe ${ }^{1}$

1. IMCA-CAT/Hauptman-Woodward Medical Research Institute, Argonne National Laboratory, Argonne IL 60439

email: mulichak@anl.gov

Regular and rapid cycles of crystallographic information are now an integral part of the pharmaceutical structure-based drug design process. The Industrial Macromolecular Crystallography Association Collaborative Access Team (IMCA-CAT) operates a macromolecular crystallography facility at the Advanced Photon Source that was founded to meet the demands of IMCA member pharmaceutical companies for reliable, high-quality, high-throughput data collection, while ensuring a secure environment for proprietary research. The 17ID micro-focused high-flux insertion device beamline, equipped with a Pilatus $6 \mathrm{M}$ pixel array detector, allows for very fast data collection times. The beam size can be easily optimized for each sample using a mini-beam quad collimator with user-selectable beam sizes of 50,20, 10 and $5 \mathrm{~mm}$. An Alio goniometer has a small $(1.2 \mu \mathrm{m})$ sphere of confusion, providing stable sample positioning, and X-ray beam position is maintained within $2 \mu \mathrm{m}$ by custom software in real time. Automated sample mounting is performed with a Rigaku ACTOR robot compatible with both Rigaku and ALS/Unipuck style magazines, providing fast yet reliable sample exchanges, and enabling remote access and unattended data collection. Mail-in is now offered as an optional access mode for IMCA members and subscribing companies, utilizing fully automated data collection for routine sample systems and manual collection by beamline staff for systems requiring more attention. A secure website provides a convenient mechanism for companies to communicate data collection requests, as well as monitor status and results for their samples. Mail-in eliminates the burden of data collection for companies and allows beamtime to be used most efficiently and flexibly. While targeting the needs of industrial research, the automation and rapid data collection times at IMCA-CAT are also well suited for structural genomics and other research efforts requiring high-throughput experiments. Access is available through subscription memberships for industrial users needing regular and guaranteed proprietary beamtime, and to academic researchers through the APS General User Program.

Keywords: synchrotron, pharmaceutical,high-throughput 